\title{
Familial Adenomatous Polyposis with Multiple Helicobacter-negative Early Gastric Cancers Treated by Endoscopic Submucosal Dissection
}

\author{
Katsuki Yaguchi ${ }^{1,2}$, Makomo Makazu ${ }^{3}$, Kingo Hirasawa ${ }^{3}$, Makoto Sugimori ${ }^{1}$, \\ Ryosuke Kobayashi ${ }^{3}$, Chiko Sato ${ }^{3}$, Ryosuke Ikeda ${ }^{3}$, Takehide Fukuchi ${ }^{3}$, Yasuaki Ishii ${ }^{3}$, \\ Hiroaki Kaneko ${ }^{1}$, Wataru Shibata ${ }^{1}$, Shoji Yamanaka ${ }^{4}$, Yoshiaki Inayama ${ }^{5}$ and Shin Maeda ${ }^{1}$
}

\begin{abstract}
:
Stomach cancer rarely develops in patients with familial adenomatous polyposis (FAP), and Helicobacter pylori infection may increase the risk of FAP-related gastric cancer. We describe the case of a 64-year-old woman who developed multiple synchronous early gastric cancers without $H$. pylori infection. Nine cancer lesions were successfully treated by endoscopic submucosal dissection. An immunohistochemical analysis revealed that the tumors were positive for mucin (MUC)2, MUC6, and CDX2, but negative for MUC5AC, suggesting that the tumors were gastrointestinal mixed type. Periodical endoscopic surveillance is important for the detection of cancers at an early stage.
\end{abstract}

Key words: familial adenomatous polyposis, endoscopic submucosal dissection, gastric cancer

(Intern Med 56: 3283-3286, 2017)

(DOI: 10.2169/internalmedicine.8735-16)

\section{Introduction}

Familial adenomatous polyposis (FAP) is an autosomal dominant hereditary disease caused by adenomatous polyposis coli (APC) mutations $(1,2)$. FAP patients have multiple colorectal adenomatous polyps. However, extracolonic neoplastic lesions are also reported in FAP patients, especially gastric neoplasms $(3,4)$. Gastric neoplasms-usually fundic gland polyps (FGP) in the gastric body-are commonly found in FAP patients in Western countries. Gastric adenoma (rarely adenocarcinoma) accounts for approximately $10 \%$ of gastric neoplasms in FAP patients $(5,6)$. In Japan, 39\% of FAP patients develop gastric adenoma and the increased incidence of adenoma may be associated with Helicobacter pylori infection $(4,7)$. Few histological analyses of FAP-associated gastric lesions have been performed. FGP and foveolar-type gastric adenoma were frequently identified; the lesions were found to be MUC5AC-positive (gastric mucin) and MUC2- and CDX2-negative (intestinal markers) (3). Intestinal gastric cancers are closely associated with $H$. pylori-related gastric atrophy and intestinal metaplasia (8). We herein report the case of a patient with FAP who developed nine synchronous early gastric cancers without $H$. pylori infection who was successfully treated by endoscopic submucosal dissection (ESD). Interestingly, a histological analysis showed that the lesions were gastrointestinal mixedtype tumors.

\section{Case Report}

The patient was a 64-year-old woman who had been diagnosed with FAP and who had undergone total colectomy at 24 years of age. She had a family history of FAP (her mother and a brother). At 64 years of age, follow-up endoscopy revealed multiple O-IIa+IIc (Paris classification) le-

\footnotetext{
${ }^{1}$ Department of Gastroenterology, Yokohama City University Graduate School of Medicine, Japan, ${ }^{2}$ Yokohama City University School of Medicine, Japan, ${ }^{3}$ Gastroenterological Centre, Yokohama City University Medical Center, Japan, ${ }^{4}$ Department of Pathology, Yokohama City University Graduate School of Medicine, Japan and ${ }^{5}$ Department of Diagnostic Pathology, Yokohama City University Medical Center, Japan Received: December 18, 2016; Accepted: April 19, 2017; Advance Publication by J-STAGE: October 11, 2017 Correspondence to Dr. Shin Maeda, shinmaeda2-gi@umin.ac.jp
} 


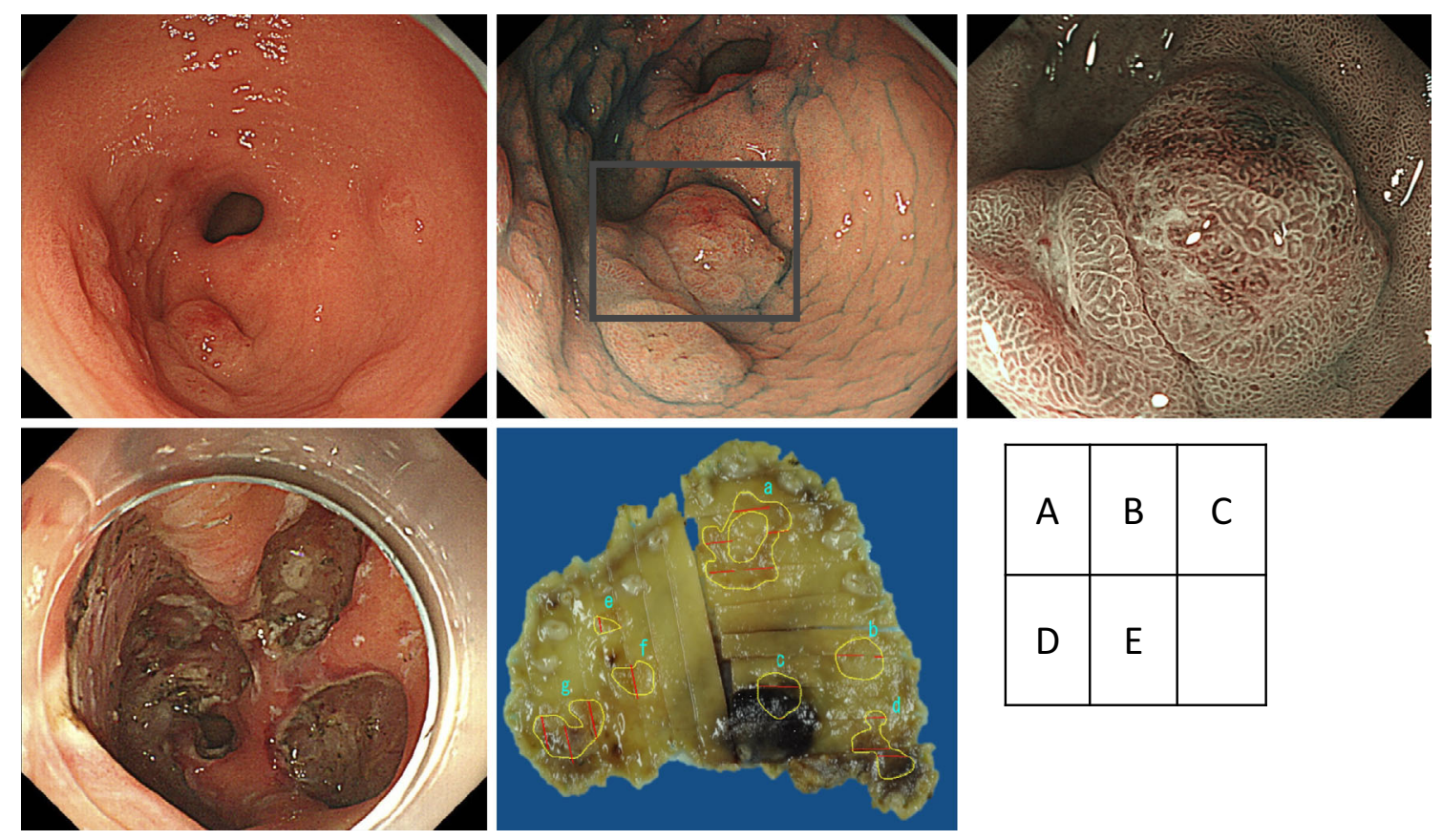

Figure 1. Endoscopic white light imaging of the tumors (A). A chromoendoscopic image of the tumors (B). Magnified NBI of a tumor (C). An endoscopic white light image after ESD (D). A formalinfixed ESD sample of the largest lesion. Red lines indicate the lesions with cancer $(E)$.

sions in the antrum of the stomach (Fig. 1A). Chromoendoscopy with indigo carmine and narrow band imaging (NBI) clearly showed a tumor area (Fig. 1B). Magnifying endoscopy with NBI (ME-NBI) indicated that the microsurface pattern of the lesions was slightly irregular and the microvascular pattern was irregular with demarcation lines. Thus, early gastric cancer was diagnosed (Fig. 1C) (9). We identified nine lesions, all of which were removed in 3 separate ESD sessions (Fig. 1D). Fig. 1E shows the largest formalin-fixed ESD sample, which included 7 adnocarninomas. The largest cancer lesion was $10 \mathrm{~mm} \times 7 \mathrm{~mm}$ in size (Fig. 1E).

A pathological analysis revealed that all nine lesions had been removed and that all of the tumors were welldifferentiated intramucosal adenocarcinomas (tub1) (Fig. 2). No venous or lymphoid vessel invasion was apparent and the treatment of the cancers was pathologically curative. The surrounding non-tumorous mucosa was almost normal without inflammation or atrophy with a regular arrangement of collecting venules, an endoscopic marker for the H. pylorinegative gastric mucosa. In accordance with the endoscopic findings, no $H$. pylori infection was identified in the pathological analysis or with serum Hp-IgG. In an immunohistochemical analysis, we used MUC5AC as a marker for foveolar cells, MUC6 for mucous neck or pyloric gland cells, MUC2 for goblet cells, CD10 for small-intestinal-type brush border, and $\mathrm{CDX} 2$ for intestinal cells. The tumors were positive for MUC6, but negative for MUC5AC. Interestingly, the tumors were also positive for MUC2, CD10, and CDX2, suggesting that they were the gastrointestinal mixed type (Fig. 2). The proliferative activity and malignant poten- tial were analyzed according to the expression of Ki-67 and TP53, which indicated that the tumors were highly proliferative and had malignant potential. In addition, $\beta$-catenin nuclear staining, which is associated with the APC mutation, was strongly positive. To prevent gastric stenosis after large ESD, triamcinolone acetonide $(50 \mathrm{mg}$ ) was injected into the ESD ulcer bed, and oral steroids were administered for 3 months. After 6 months, the ESD ulcer had healed without stenosis.

\section{Discussion}

There are a few reports on the gastric lesions in FAP patients. The reported rate of FAP-associated gastric cancer in Japan is higher than that in Western countries. In Japan, gastric cancer developed in 11 of 261 FAP patients (4.2\%) (10), whereas it was found that the risk of gastric cancer did not differ between FAP and non-FAP patients in Western countries (11). This difference may be due to the higher rate of gastric atrophy or intestinal metaplasia in the Japanese population, which is associated with a higher rate of $H$. pylori infection (4). However, H. pylori infection and gastric atrophy were not found in this case. Another reason for this difference is that there are probably differences between Western and Asian FAP patients in the mutation loci in the APC gene (12). We have not yet analyzed the APC mutation locus in the current case.

The histology of FAP-related gastric cancer has not been clarified. In FAP patients, fundic gland polyps (FGP) are frequently observed and may be precursor lesions of gastric adenocarcinoma (13). In this context, FGP-derived cancer 


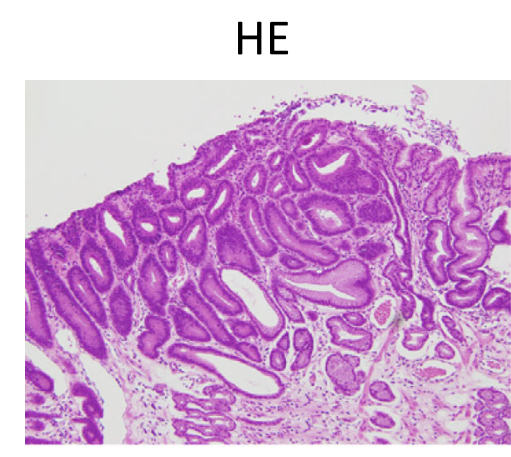

MUC6

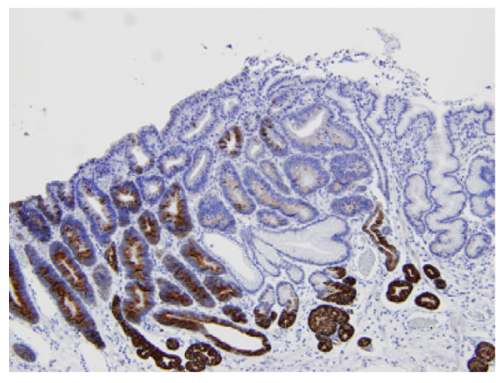

Ki67

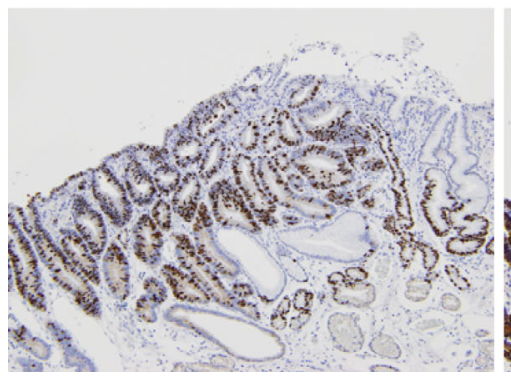

MUC2

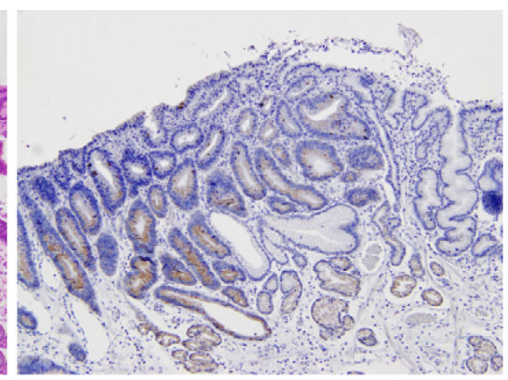

CDX2

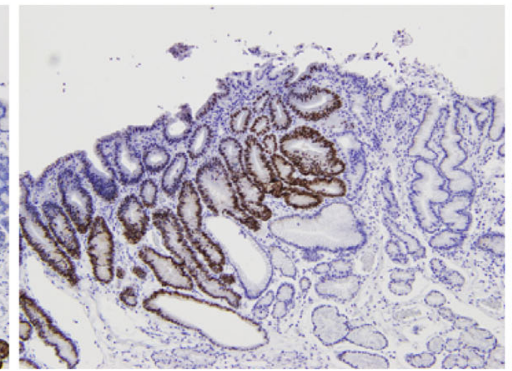

$\beta$-catenin
MUC5AC

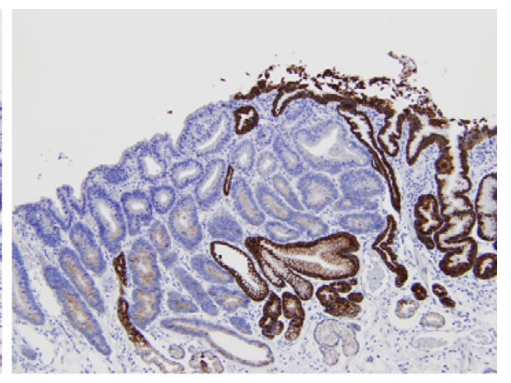

CD10

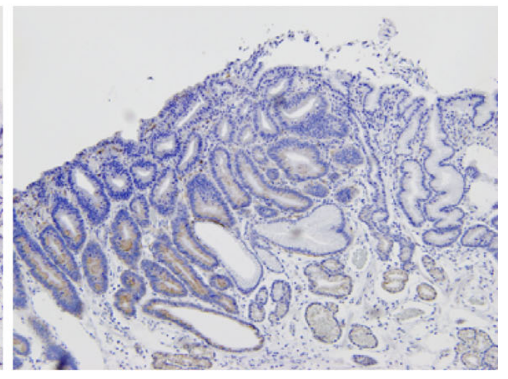

TP53

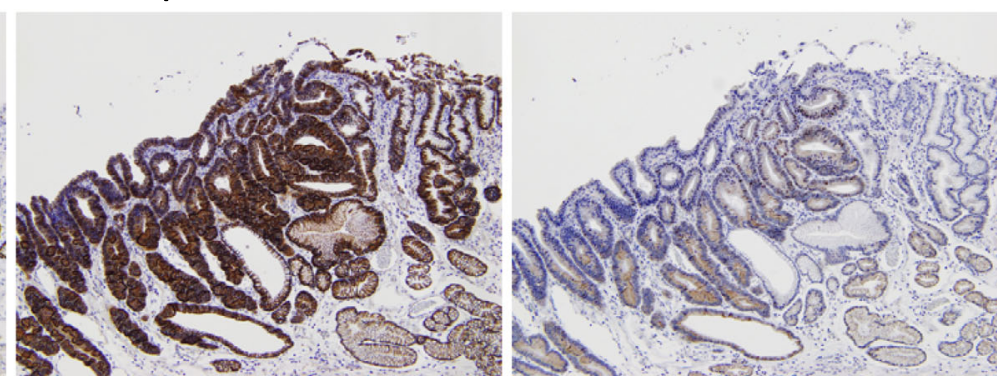

Figure 2. Hematoxylin and Eosin staining and immunohistochemical staining for MUC2, MU-

C5AC, MUC6, CDX2, CD10, Ki67, $\beta$-catenin, and TP53. Original $\times 40$.

might be positive for MUC5AC, which is a marker for gastric foveolar cells. Hashimoto et al. reported that, in addition to FGP, pyloric gland adenoma (PGA) was frequently observed in FAP patients and was strongly positive for MUC5AC and MUC6 (14). However, FGP and PGA were negative for MUC2 and CDX2 when not accompanied by intestinal metaplasia (15). In our case, the tumors were positive for MUC2, CDX2, and MUC6, but negative for MUC5AC, suggesting that the lesions were gastrointestinal mixed type. The background mucosa did not show inflammation, $H$. pylori infection, or intestinal metaplasia, making the tumors in this case an unusual gastric cancer phenotype. Mutations of the APC gene cause the accumulation of $\beta$ catenin and might be one of the mechanisms responsible for the development of gastric cancer in FAP patients (14). The overexpression of CDX2 in gastric epithelial cells might induce intestinal metaplasia and eventually cause adenocarcinoma (16), suggesting that, in addition to the accumulation of $\beta$-catenin, the overexpression of CDX2 is one of the mechanisms underlying the development of gastric adenocarcinoma. This assumes that additional genetic or epigenetic alterations cause the accumulation of CDX2. Further studies should be performed to analyze the mechanisms of these alterations.

It is questionable whether the adenocarcinomas in the current case were developed as a result of the adenomacarcinoma sequence or whether they occurred as de novo cancer. We tried to analyze a sample of the adenoma, which had been resected by ESD 7 years previously and found that the tumor was positive for MUC2 and CDX2, but negative for MUC5AC and MUC6, suggesting that the adenoma was of the intestinal type. We also found that $\beta$-catenin was weakly stained and TP53 nuclear staining was negative (Supplementary Figure). Taken together, it is likely that the adenocarcinoma in the current case was caused via the adenoma-carcinoma sequence, as is the case of colon cancer.

Nine synchronous gastric cancers were detected in the current case. The reported frequency of synchronous gastric cancer is approximately $10 \%$; cases involving more than five synchronous lesions are very rare (17). Shibata et al. reported that three of five FAP patients with gastric cancer had synchronous cancers, indicating that the gastric cancers in FAP patients tend to be synchronous. In addition, metachronous gastric cancer has been reported in FAP pa- 
tients (18); thus, it is important to perform periodic endoscopic surveillance to diagnose cancers at an early stage. Our patient had undergone ESD for gastric adenoma 7 years previously, and multiple cancers were found after careful EGD surveillance.

The authors state that they have no Conflict of Interest (COI).

\section{References}

1. Groden J, Thliveris A, Samowitz W, et al. Identification and characterization of the familial adenomatous polyposis coli gene. Cell 66: 589-600, 1991.

2. Kinzler KW, Nilbert MC, Su L-K, et al. Identification of FAP locus genes from chromosome 5q21. Science 253: 661-665, 1991.

3. Wood LD, Salaria SN, Cruise MW, et al. Upper GI tract lesions in familial adenomatous polyposis (FAP): enrichment of pyloric gland adenomas and other gastric and duodenal neoplasms. Am J Surg Pathol 38: 389-393, 2014.

4. Nakamura S, Matsumoto T, Kobori Y, Iida M. Impact of Helicobacter pylori infection and mucosal atrophy on gastric lesions in patients with familial adenomatous polyposis. Gut 51: 485-489, 2002.

5. Sarre RG, Frost AG, Jagelman DG, et al. Gastric and duodenal polyps in familial adenomatous polyposis: a prospective study of the nature and prevalence of upper gastrointestinal polyps. Gut 28: 306-314, 1987.

6. Spigelman AD, Williams CB, Talbot IC, et al. Upper gastrointestinal cancer in patients with familial adenomatous polyposis. Lancet 2: 783-785, 1989.

7. Iwama T, Mishima Y, Utsunomiya J. The impact of familial adenomatous polyposis on the tumorigenesis and mortality at the several organs. Its rational treatment. Ann Surg 217: 101-108, 1993.

8. Uemura N, Okamoto S, Yamamoto S, et al. Helicobacter pylori infection and the development of gastric cancer. N Engl J Med
345: 784-789, 2001.

9. Yao K. The endoscopic diagnosis of early gastric cancer. Ann Gastroenterol 26: 11-22, 2013.

10. Yamaguchi T, Ishida $\mathrm{H}$, Ueno $\mathrm{H}$, et al. Upper gastrointestinal tumours in Japanese familial adenomatous polyposis patients. Jpn $\mathrm{J}$ Clin Oncol 46: 310-315, 2016.

11. Offerhaus GJ, Giardiello FM, Krush AJ, et al. The risk of upper gastrointestinal cancer in familial adenomatous polyposis. Gastroenterology 102: 1980-1982, 1992.

12. Miyaki M, Yamaguchi T, Iijima T, et al. Difference in characteristics of APC mutations between colonic and extracolonic tumors of FAP patients: variations with phenotype. Int J Cancer 122: 24912497, 2008.

13. Garrean S, Hering J, Saied A, et al. Gastric adenocarcinoma arising from fundic gland polyps in a patient with familial adenomatous polyposis syndrome. Am Surg 74: 79-83, 2008.

14. Hashimoto T, Ogawa R, Matsubara A, et al. Familial adenomatous polyposis-associated and sporadic pyloric gland adenomas of the upper gastrointestinal tract share common genetic features. Histopathology 67: 689-698, 2015.

15. Chen ZM, Scudiere JR, Abraham SC, Montgomery E. Pyloric gland adenoma: an entity distinct from gastric foveolar type adenoma. Am J Surg Pathol 33: 186-193, 2009.

16. Mutoh H, Sakurai S, Satoh K, et al. Development of gastric carcinoma from intestinal metaplasia in Cdx2-transgenic mice. Cancer Res 64: 7740-7747, 2004.

17. Isobe T, Hashimoto K, Kizaki J, et al. Characteristics and prognosis of synchronous multiple early gastric cancer. World J Gastroenterol 19: 7154-7159, 2013.

18. Shibata C, Ogawa H, Miura K, et al. Clinical characteristics of gastric cancer in patients with familial adenomatous polyposis. Tohoku J Exp Med 229: 143-146, 2013.

The Internal Medicine is an Open Access article distributed under the Creative Commons Attribution-NonCommercial-NoDerivatives 4.0 International License. To view the details of this license, please visit (https://creativecommons.org/licenses/ by-nc-nd/4.0/).

(C) 2017 The Japanese Society of Internal Medicine Intern Med 56: 3283-3286, 2017 